\title{
MULTILOCUS SEQUENCE TYPING (MLST) BASED GENETIC VARIATION ANALYSIS OF SHISHAM DIEBACK ASSOCIATED STRAINS OF CERATOCYSTIS FIMBRIATA SENSU LATO SPECIES COMPLEX IN PAKISTAN
}

\author{
UL HAQ, I. ${ }^{1 *}-$ IJAZ, S. $^{2}-$ LATIF, M. Z. ${ }^{1}$ \\ ${ }^{I}$ Department of Plant Pathology, University of Agriculture, Faisalabad, Pakistan \\ ${ }^{2}$ Centre of Agricultural Biochemistry and Biotechnology (CABB), University of Agriculture, \\ Faisalabad, Pakistan \\ *Corresponding author \\ e-mail: imran_1614@yahoo.com; phone: +92-34-4445-3063
}

(Received $17^{\text {th }}$ May 2019; accepted $16^{\text {th }}$ Jul 2019)

\begin{abstract}
Ceratocystis fimbriata sensu lato species complex is a group of highly diverse cryptic species known to be associated with different diseases in a range of host plants. For the accurate identification of fungal pathogens belonging to this species complex, molecular approaches have been employed in juxtaposition with morphological characterization. Different DNA based methods have become the routine practice of mycologist community throughout the world. Among them, multilocus sequence typing (MLST) approach is gaining popularity. Four fungal taxonomy groups have been established MLST scheme for few fungi, to date. However, in Ceratocystis fimbriata species complex, no report on MLST based genotyping is available yet. Therefore, by considering this perspective, we attempted MLST method for genotyping and diversity analysis of this species complex. We investigated ITS and TEF1- $\alpha$ loci, for two multilocus sequence typing scheme. Allelic profile of each locus gave 23 sequence types (STs) and 22 Allele types (ATs). However, the typing efficiency of ITS region was high as compared to TEF1- $\alpha$. The phylogenetic network parallelograms based on neighbor net algorithm also showed genetic diversity in studied isolates. This relationship study was further supported by generating phylogram, which resolved these isolates in three different clades showing unequivocal phylogenetic diversity and relatedness.
\end{abstract}

Keywords: Ceratocystis fimbriata, Dalbergia sissoo, genotyping variation, sequence types (STs), allele types (ATs)

\section{Introduction}

Ceratocystis fimbriata complex represents a group of cryptic species of sever plant pathogen(s) and saprobe(s) as well (Wyk, 2005). C. fimbriata complex parasitizes a wide range of plant families from herbaceous to woody plants. The $C$. fimbriata was first time reported in Sweet potato in 1890 (Halsted, 1890). Since 1980, several fungi of species were isolated and identified from a range of host plants from around the globe (Seifert et al., 1993; Wyk et al., 2005). C. fimbriata is widely accepted as a complex that comprised of Latin American, North American, Asian and the African clades that include many fungal species (Johnson et al., 2005; Heath et al., 2009). In past few decades, new taxa have been evolved extensively in this complex with special reference to advancement in DNA based identification methods (Fourie et al., 2015). Different Ceratocystis species in the $C$. fimbriata sensu lato species complex have been reported to date that are associated with different plant diseases (Harrington, 2000). The fungi of this species complex causes root rot, cankers and vascular wilts and staining in woody hosts (Wingfield et al., 1996; Marin et al., 2003; Al Adawi et al., 2013). 
The pathogens belong to Ceratocystis fimbriata sensu lato species complex are soil borne with fruity odor characteristics. This feature facilitates their dispersal by insects and beetles (Wyk et al., 2005). With wide host rang, this species complex has been recognized as of diverse taxonomic entity. However, with the aid of molecular based studies, different taxa have been recognized and diverged as distinct species from this species complex. It has been documented by DNA based studies that Ceratocystis fimbriata species complex represents diverse and discrete grouping based on geography and host (Harrington, 2000; Baker et al., 2003). Previously, in this complex, species delineation was made based on single gene genealogy of ITS (internal transcribed spacer) region. Nevertheless, multiple gene genealogies and analyzing phylogenetic diversity had been attempted to specify species boundaries.

However, there are diverse molecular tools employing for genotypic diversity analysis. Whereas, multilocus sequence typing (MLST) tool has become more popular scheme for genetic diversity studies. In fungal molecular biology, so far established MLST schemes are for Fusarium species (Westerdijk Fungal Biodiversity Institute), Candida species (Imperial College), Aspergillus (Oxford University), Bipolaris species and Scedosporium apiospermum and S. boydii species (International Society of Human and Animal Mycology, ISHAM). However, in Ceratocystis species complex, MLST scheme has not been established yet. Although this complex is a cryptic species complex and there is a great room of genetic diversity. By considering this perspective, we have attempted two multilocus sequence typing (MLST) method for genotyping and analyzing genetic variation in Ceratocystis species complex. This may be used for further studies based on other loci. Hence, the data generated by MLST scheme could be used for epidemiological studies for which accurate recognition of different species, subspecies and strains are required.

\section{Material and methods}

\section{Fungal isolates}

We used 23 isolates of Ceratocystis fimbriata sensu lato from our collection of fungal isolates associated to dieback affected shisham (Dalbergia sissoo) plants. Each isolate was characterized based on its morphocultural characteristics and was labelled as FMB (Fungal Molecular Biology Laboratory-Culture Collection) with a unique identification number as given in Table 1.

\section{DNA extraction}

Fungal DNA was extracted with GeneJET Genomic DNA Purification Kit (Thermoscientific, USA). The DNA samples were quantified with a NanoDrop 8000 spectrophotometer (Thermofisher). However, DNA quality was checked on agarose gel electrophoresis and stored at $-20{ }^{\circ} \mathrm{C}$ until further use.

\section{Molecular biology and computational biology}

We used two genes, internal transcribed spacer (ITS) and translation elongation factor $1 \alpha(\mathrm{TEF} 1-\alpha)$, for polymerase chain reaction (PCR), sequencing and phylogenetic analysis. PCR amplifications of these two genetic regions was carried out in Veriti ${ }^{\mathrm{TM}} 96$ wells thermalcycler (Applied Biosystems) using $25 \mu \mathrm{l}$ reaction volume with the ITS (White et al., 1990) and TEF1- $\alpha$ (Oliveira et al., 2015; Li et al., 2017) sequence regions 
based primer pairs. PCR products were purified using a FavorPrep PCR purification kit (Favorgen Biotech Corporation, Taiwan) and were sequenced in both directions (Eurofins Genomics DNA sequencing services, USA). High quality trimmed sequences were generated using BioEdit software version 7.2.6.1. For getting consensus sequences of sequences obtained using forward and reverse primers DNASTAR Lasergene v. 7.1.0 SeqMan pro (SeqManTMII) software package was used. These generated sequences were compared with the sequences available in NCBI database using BLASTn and were deposited in GenBank under accession numbers, given in the Table 1.

Table 1. A list of isolates name, strain accession number and GenBank accession numbers of sequences of ITS and TEF1- $\alpha$

\begin{tabular}{c|c|c|c|c|c}
\hline Sr \# & Isolate name & $\begin{array}{c}\text { Strain accession } \\
\text { number }\end{array}$ & Location & \multicolumn{2}{|c}{$\begin{array}{c}\text { GenBank accession } \\
\text { numbers }\end{array}$} \\
\cline { 5 - 6 } & & & ITS & TEF1-a \\
\hline 1 & FMB-GHP1E-SD & FMB 0146 & Punjab, Pakistan & MK816923 & MK890310 \\
2 & FMB-GHP2E-SD & FMB 0147 & Punjab, Pakistan & MK816925 & MK890311 \\
3 & FMB-GHP3-SD & FMB 0148 & Punjab, Pakistan & MK816928 & MK890312 \\
4 & FMB-GHP4-SD & FMB 0149 & Punjab, Pakistan & MK816929 & MK890313 \\
5 & FMB-GHP5-SD & FMB 0150 & Punjab, Pakistan & MK816932 & MK890314 \\
6 & FMB-GHP6-SD & FMB 0160 & Punjab, Pakistan & MK816933 & MK890315 \\
7 & FMB-GHP7-SD & FMB 0161 & Punjab, Pakistan & MK816934 & MK890316 \\
8 & FMB-GHP8-SD & FMB 0162 & Punjab, Pakistan & MK816957 & MK890317 \\
9 & FMB-M12-SD & FMB 0033 & Punjab, Pakistan & MF767443 & MK890321 \\
10 & FMB-BWP-SD & FMB 0163 & Punjab, Pakistan & MK811131 & MK820654 \\
11 & FMB-VR-SD & FMB 0164 & Punjab, Pakistan & MK813838 & MK890320 \\
12 & FMB-ST-SD & FMB 0165 & Punjab, Pakistan & MK813839 & MK890322 \\
13 & FMB-BR-SD & FMB 0166 & Punjab, Pakistan & MK811133 & MK820656 \\
14 & FMB-FSD-SD & FMB 0167 & Punjab, Pakistan & MK811134 & MK850855 \\
15 & FMB-TTS-SD & FMB 0168 & Punjab, Pakistan & MK811125 & MK890319 \\
16 & FMB-BLCN-01 & FMB 0169 & Baluchistan, Pakistan & MK811095 & MK850853 \\
17 & FMB-BLCN-02 & FMB 0170 & Baluchistan, Pakistan & MK811097 & MK820655 \\
18 & FMB-Thatta-SD & FMB 0171 & Sindh, Pakistan & MK811092 & MK850852 \\
19 & FMB-Hyderabad-SD & FMB 0172 & Sindh, Pakistan & MK811093 & MK850851 \\
20 & FMB-Nosheroferoz-SD & FMB 0173 & Sindh, Pakistan & MK811094 & MK850850 \\
21 & FMB-GL-SD & FMB 0174 & KPK, Pakistan & MK811103 & MK890318 \\
22 & FMB-BZ1-SD & FMB 0175 & KPK, Pakistan & MK811102 & MK910747 \\
23 & FMB-PZ1-SD & FMB 0176 & KPK, Pakistan & MK811101 & MK850854 \\
\hline
\end{tabular}

\section{Genotyping variation analysis of ITS and TEF1- $\alpha$ genes}

Nucleotide sequences of ITS and TEF1- $\alpha$ genes were used for genetic distance analysis. Genotypic variation of each locus was assessed using MLSTest v1.0 software. The allele types (ATs) number, sequence types (STs) number, number of polymorphism, typing efficiency and discrimination power at $95 \%$ confidence interval were computed using this software. Neighbor net algorithm was used to perform phylogenetic network of each loci by splitsTree4 V4.14.8 software. 
Nucleotide sequences of both genes were concatenated using Geneious software v. 4.8.5 in the order as ITS and TEF1- $\alpha$ to generate concatenated dataset file. The best evolutionary model for concatenated dataset was selected from Bayesian Information Criterion (BIC) using MEGA 7.0 software. However, evolutionary model with lowest BIC value was selected to construct a maximum likelihood phylogram. Sequences were aligned with ClustalW for phylogenetic tree construction. A phylogenetic tree of the 23 aligned sequences based on best evolutionary model was obtained for the heuristic search in bootstrap analysis with 1000 replications by applying the Neighbor-Joining method to a matrix of pairwise distances estimated through Maximum Composite Likelihood (MCL) approach using MEGA7 (Molecular Evolutionary Genetics Analysis version 7.0) software.

\section{Results}

Twenty-three fungal isolates collected from dieback disease affected tissues of Dalbergia sissoo (Shisham) were characterized based on morphological and genomic investigations. These characterizations has set them under the Ceratocystis species complex. Molecular characterization was based on the direct sequencing of ITS and TEF1- $\alpha$ regions. The generated sequences of these isolates were subjected to BLASTn tool to search their homology with already available sequences of the type materials in NCBI database (https://blast.ncbi.nlm.nih.gov/Blast.cgi). The sequence similarity using BLASTn showed significant similarity with Ceratocystis species of Ceratocystis fimbriata sensu lato complex.

The Genotypic variation profiling of 23 isolates belongs to Ceratocystis fimbriata sensu lato complex was generated using MLSTest software. The analysis showed 23 best different sequence types (STs) numbers and 22 allele types (ATs) numbers of both loci (Table 2). However, the frequency of each allele types (ATs) numbers in each isolate is given in Table 3.

The allelic profile of both genes including number of alleles, discrimination power (95\% confidential interval), typing efficacy and number of polymorphisms were calculated, given in the Table 4. A diverse genetic relationship was found among the genotyped variants of 23 Ceratocystis fimbriata sensu lato after analyzing phylogenetic network of each of the both loci (ITS and TEF1- $\alpha$ ) using neighbor net algorithm (Fig. 1).

Among 24 calculated models of concatenated dataset, the best evolutionary model of concatenated dataset (ITS and TEF1- $\alpha$ ) for maximum likelihood (ML) phylogenetic tree was K2 (Kimura 2-parameter) with BIC (Bayesian Information Criterion) score 2721.116. This model was selected based on the theory that evolutionary models with the lowest BIC values are considered to describe best substitution pattern. The phylogenetic tree (Fig. 2) with highest log likelihood (-1145.9648) was drawn to scale, with branch lengths measured in the number of substitutions per site. Codon positions included were $1 \mathrm{st}+2 \mathrm{nd}+3 \mathrm{rd}+$ Noncoding. There were 749 positions in the final dataset.

The evolutionary hierarchy was inferred using ML method based on the K2 model, which categorized these fungal isolates into three discrete clades. The phylogram showed that the clade III was outer group and comprised of FMB-BZ1-SD, FMB-M12-SD, FMBThatta-SD, FMB-ST-SD, FMB-GHP2E-SD, FMB-BLCN-01, and FMB-GHP1E-SD with 96\% bootstrap support. However, Clade I and Clade II were shown to be evolutionary diverged from Clade III with $96 \%$ and $86 \%$ bootstrap support respectively (Fig. 2). 


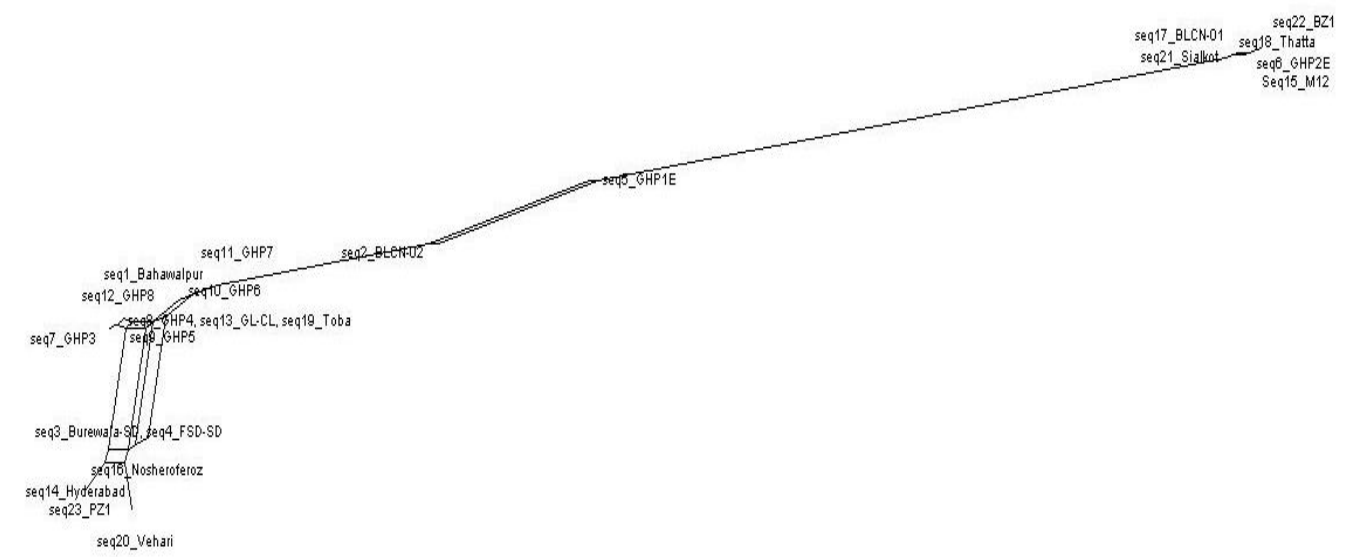

a

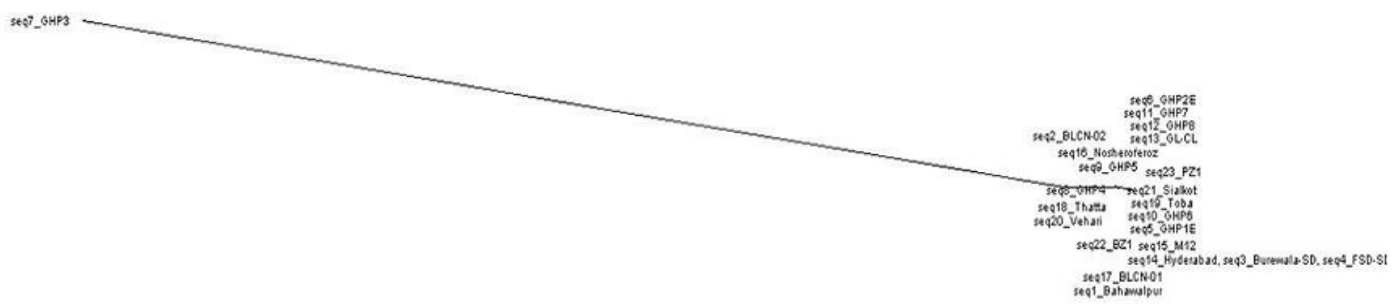

b

Figure 1. Phylogenetic network of two loci, (a) ITS, (b) TEF1- $\alpha$ with neighbor net algorithm by split tree software

Clade I majorly comprised of fungal isolates from Punjab (a province of Pakistan), as FMB-GHP3-SD, FMB-GHP4-SD, FMB-GHP5-SD, FMB-GHP6-SD, FMB-GHP7-SD, FMB-BWP-SD, and FMB-TTS-SD except FMB-BLCN-02, which was collected from Baluchistan (a province of Pakistan). These fungal isolates made cladding with $96 \%$ bootstrap support. However, in clade II fungal isolates from KPK, Sindh and Punjab (provinces of Pakistan) made grouping. In clade II, the isolate FMB-PZ1-SD (KPK) was evolutionary diverged from clade III with $86 \%$ bootstrap support. However, fungal isolates of this group, FMB-Nosheroferoz-SD, FMB-Hyderabad-SD (Sindh), FMB-BRSD, FMB-FSD-SD and FMB-VR-SD (Punjab) were shown to diverged from FMBPZ1-SD and grouped together in this clade with $67 \%$ bootstrap support (Fig. 2). Based on the phylogenetic networks of both loci and phylogenetic tree of concatenated dataset, among these 23 studied isolates, 9 isolates (FMB-BLCN-02, FMB-GHP3-SD FMBGHP7-SD, FMB-PZ1-SD, FMB-FSD-SD, FMB-Nosheroferoz-SD, FMB-BZ1-SD, FMB-M12-SD and FMB-GHP1E-SD) were selected for phylogenetic analyses to delimit the species boundaries of Ceratocystis species associated to dieback disease of Dalbergia sissoo. These selected isolates showed more genotyping variation among all studied isolates by multilocus sequence typing (MLST) approach. 
Table 2. A list of sequence types (STs) numbers and allele types (ATs) numbers of ITS and TEF1- $\alpha$ loci in isolates of Ceratocystis fimbriata sensu lato complex

\begin{tabular}{c|c|c|c|c}
\hline \multirow{2}{*}{ Isolate name } & \multirow{2}{*}{ Strain number } & \multicolumn{2}{|c|}{ Allele types (ATs) } & Sequence types (STs) \\
\cline { 3 - 5 } & TEF1-a & ITS & \\
\hline FMB-BR-SD & FMB 0166 & 1 & 1 & 1 \\
FMB-PZ1-SD & FMB 0176 & 2 & 2 & 2 \\
FMB-FSD-SD & FMB 0167 & 3 & 3 & 3 \\
FMB-Nosheroferoz-SD & FMB 0173 & 4 & 4 & 4 \\
FMB-Hyderabad-SD & FMB 0172 & 5 & 5 & 5 \\
FMB-VR-SD & FMB 0164 & 6 & 5 & 6 \\
FMB-BLCN-02 & FMB 0170 & 7 & 6 & 7 \\
FMB-GHP8-SD & FMB 0162 & 8 & 7 & 8 \\
FMB-GHP5-SD & FMB 0150 & 9 & 8 & 9 \\
FMB-GHP4-SD & FMB 0149 & 10 & 9 & 10 \\
FMB-GL-SD & FMB 0174 & 7 & 10 & 11 \\
FMB-BWP-SD & FMB 0163 & 11 & 11 & 12 \\
FMB-GHP7-SD & FMB 0161 & 12 & 12 & 13 \\
FMB-GHP3-SD & FMB 0148 & 13 & 13 & 14 \\
FMB-GHP6-SD & FMB 0160 & 14 & 14 & 15 \\
FMB-TTS-SD & FMB 0168 & 15 & 15 & 16 \\
FMB-GHP2E-SD & FMB 0147 & 16 & 16 & 17 \\
FMB-BLCN-01 & FMB 0169 & 17 & 17 & 18 \\
FMB-M12-SD & FMB 0033 & 18 & 18 & 19 \\
FMB-BZ1-SD & FMB 0175 & 19 & 19 & 20 \\
FMB-Thatta-SD & FMB 0171 & 20 & 20 & 21 \\
FMB-ST-SD & FMB 0165 & 21 & 21 & 22 \\
FMB-GHP1E-SD & FMB 0146 & 22 & 22 & 23 \\
\hline
\end{tabular}

Pertaining to allelic profile for multilocus sequence typing (MLST), typing efficiency of ITS locus was more (0.124) than TEF1- $\alpha$ (0.021). This genotyping supports the phylogram generated through concatenated dataset of both loci. This genotyping results have deduced that, the ITS region has more resolving and typing power than TEF1- $\alpha$ region to species identification and discrimination in case of Ceratocystis species of Ceratocystis fimbriata sensu lato complex. This is the first report of using two multilocus sequence typing (MLST) scheme for studying and typing genetic variation in Ceratocystis species complex.

\section{Discussion}

There are different approaches that have been employing for genotypic diversity studies. Among of them, multilocus sequence typing (MLST) has become more robust scheme. In the field of fungal molecular biology, MLST scheme Fusarium species, Candida species, Aspergillus, Bipolaris species and Scedosporium apiospermum and S. boydii species have been established by four working groups of Fungal taxonomy. However, in Ceratocystis species complex, MLST scheme has not been established yet. Although this complex is a cryptic species complex and there is a great room of genetic diversity. By considering this perspective, we have attempted two multilocus sequence typing (MLST) method in Ceratocystis species complex. 


$$
-12579 \text { - }
$$

Table 3. A list of allele types (ATs) numbers of ITS and TEF1- $\alpha$ with their frequency in each isolate

\begin{tabular}{|c|c|c|c|}
\hline Gene & ATs & Frequency & Isolate name \\
\hline \multirow{22}{*}{ TEF1- $\alpha$} & 1 & 1 & FMB-BR-SD \\
\hline & 2 & 1 & FMB-PZ1-SD \\
\hline & 3 & 1 & FMB-FSD-SD \\
\hline & 4 & 1 & FMB-Nosheroferoz-SD \\
\hline & 5 & 1 & FMB-Hyderabad-SD \\
\hline & 6 & 1 & FMB-VR-SD \\
\hline & 7 & 2 & FMB-GL-SD, FMB-BLCN-02 \\
\hline & 8 & 1 & FMB-GHP8-SD \\
\hline & 9 & 1 & FMB- GHP5-SD \\
\hline & 10 & 1 & FMB-GHP4-SD \\
\hline & 11 & 1 & FMB-BWP-SD \\
\hline & 12 & 1 & FMB-GHP7-SD \\
\hline & 13 & 1 & FMB-GHP3-SD \\
\hline & 14 & 1 & FMB-GHP6-SD \\
\hline & 15 & 1 & FMB-TTS-SD \\
\hline & 16 & 1 & FMB-GHP2E-SD \\
\hline & 17 & 1 & FMB-BLCN-01 \\
\hline & 18 & 1 & FMB-M12-SD \\
\hline & 19 & 1 & FMB-BZ1-SD \\
\hline & 20 & 1 & FMB-Thatta-SD \\
\hline & 21 & 1 & FMB-ST-SD \\
\hline & 22 & 1 & FMB-GHP1E-SD \\
\hline \multirow{22}{*}{ ITS } & 1 & 1 & FMB-BR-SD \\
\hline & 2 & 1 & FMB-PZ1-SD \\
\hline & 3 & 1 & FMB-FSD-SD \\
\hline & 4 & 1 & FMB-Nosheroferoz-SD \\
\hline & 5 & 2 & FMB-Hyderabad-SD, FMB-VR-SD \\
\hline & 6 & 1 & FMB-BLCN-02 \\
\hline & 7 & 1 & FMB-GHP8-SD \\
\hline & 8 & 1 & FMB- GHP5-SD \\
\hline & 9 & 1 & FMB-GHP4-SD \\
\hline & 10 & 1 & FMB-GL-SD \\
\hline & 11 & 1 & FMB-BWP-SD \\
\hline & 12 & 1 & FMB-GHP7-SD \\
\hline & 13 & 1 & FMB-GHP3-SD \\
\hline & 14 & 1 & FMB-GHP6-SD \\
\hline & 15 & 1 & FMB-TTS-SD \\
\hline & 16 & 1 & FMB-GHP2E-SD \\
\hline & 17 & 1 & FMB-BLCN-01 \\
\hline & 18 & 1 & FMB-M12-SD \\
\hline & 19 & 1 & FMB-BZ1-SD \\
\hline & 20 & 1 & FMB-Thatta-SD \\
\hline & 21 & 1 & FMB-ST-SD \\
\hline & 22 & 1 & FMB-GHP1E-SD \\
\hline
\end{tabular}




$$
-12580-
$$

Table 4. Allelic profile including number of polymorphism, number of alleles, typing efficiency, discriminatory power of ITS and TEF1- $\alpha$ genes

\begin{tabular}{c|c|c|c|c}
\hline Locus & No. of alleles & No. of polymorphisms & Typing efficiency & $\begin{array}{c}\text { Discrimination power } \\
\text { (95\% confidence } \\
\text { interval) }\end{array}$ \\
\hline ITS & 22 & 177 & 0.124 & $0.996(0.985-1)$ \\
TEF1- $\alpha$ & 22 & 1049 & 0.021 & $0.996(0.985-1)$ \\
\hline
\end{tabular}

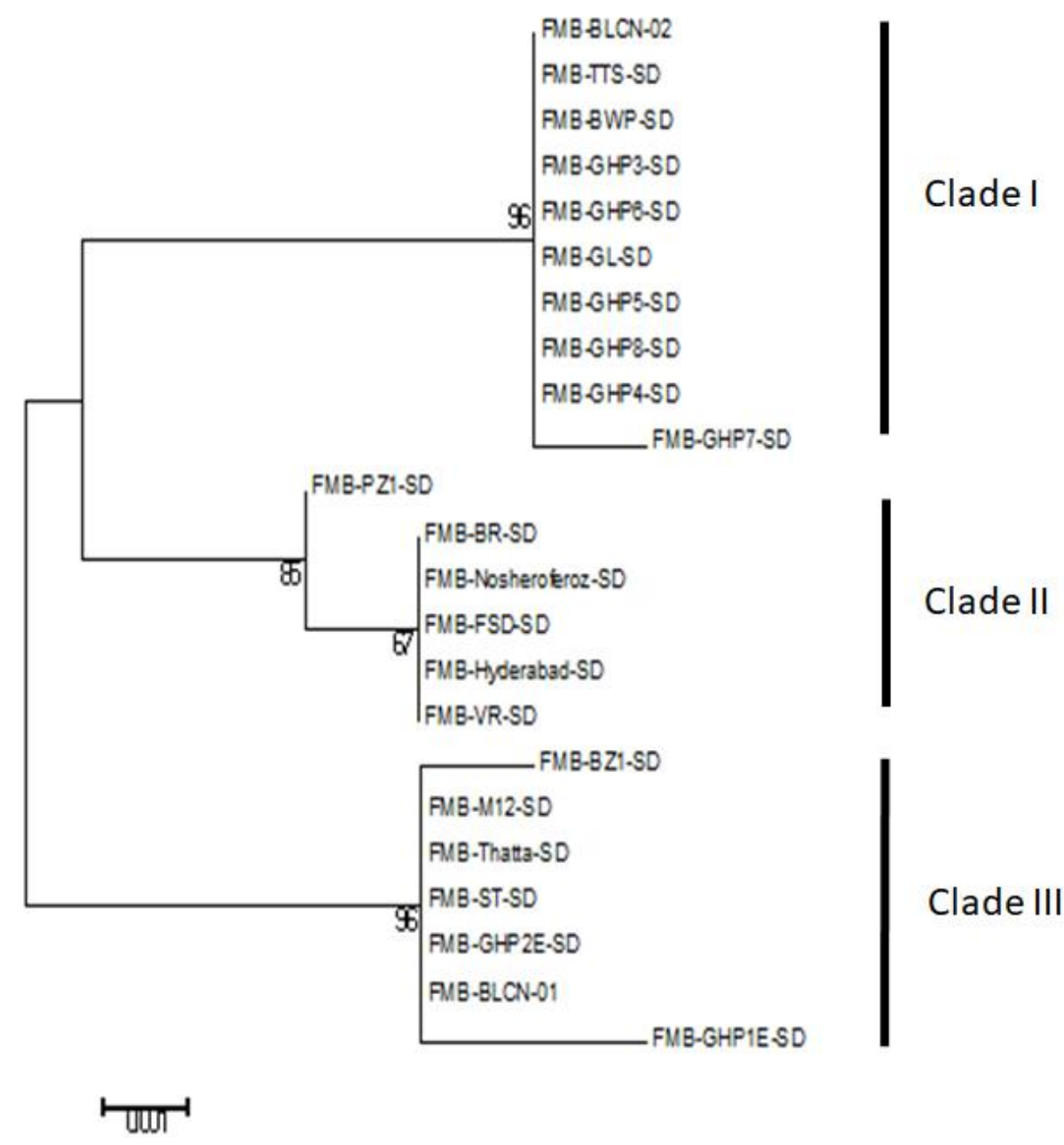

Figure 2. A maximum likelihood (ML) phylogenetic tree of concatenated dataset of two loci (ITS and TEF1- $\alpha$ ) based on evolutionary model K2 (Kimura 2-parameter)

We have attempted to study the genetic variation and typing analysis among the isolates of Ceratocystis species. We have 23 isolates of this fungus collected from dieback tissues of Dalbergia sissoo. Initially we have used 2 loci, internal transcribed spacer (ITS) and translation elongation factor $1 \alpha$ (TEF1- $\alpha$ ), for MLST based genotyping analysis of Ceratocystis fimbriata sensu lato species complex. Successful sequencing of both loci in all isolates was attempted. We identified 23 STs (sequence types) in both loci. The number of polymorphism for each locus was varied which was 177 for ITS and 1049 for TEF1- $\alpha$. 
The sequences of 23 isolates of Ceratocystis fimbriata sensu lato species complex were analyzed by applying neighbor net algorithm using Splits-Tree software. The graph of ITS region-based sequences generated by Splits-Tree was shown as parallelogram, which implied all could be diverse but characters are shared by a set of species. However, the graph of TEF1- $\alpha$ showed the clustering of species at the one end of graphical string except FMB-GHP3-SD on its other end. The genetic network not shown to be able to describe genetic variation. This phylogenetic network results supported the allelic profiles generated by MLST tool which showed typing efficiency of ITS is more (0.124) as compared to TEF1- $\alpha(0.021)$, however, their discriminatory power was same (0.996). The sequence types (STs) number were 23 in case of both loci, which depicted that each isolates had one ST type. However, the allele types (ATs) number was 22 in both loci. Each isolates has one type of allele except FMB-GL-SD, FMB-BLCN-02 that have same type of allele of TEF1- $\alpha$. Similarly, in case of ITS loci, each isolate has one type of allele except FMB-Hyderabad-SD, FMB-VR-SD that possess same type of allele.

In nutshell, here we have presented first two multilocus sequence typing (MLST) scheme based relationship analysis among Ceratocystis fimbriata sensu lato species complex associated to dieback disease in Dalbergia sissoo in Pakistan to studying and typing genetic variation in Ceratocystis species complex. In future, more loci may be explored for allelic profiling of these fungal isolates. Allele types (ATs) of those loci could be appraised to apprise sequence types (STs) numbers of these fungal isolates in way that is more precise.

\section{Conclusion}

Ceratocystis fimbriata sensu lato species complex is a group of cryptic species. Several species have been evolved and identified as discrete taxa. In Ceratocystis fimbriata species complex, no report on MLST based genotyping is available yet. We investigated two loci (ITS and TEF1- $\alpha$ ) of 23 fungal isolates from dieback disease affected tissues of Dalbergia sissoo for MLST scheme. In allelic profile, the typing efficiency of ITS region was shown to be high by scoring the value of 0.124 as compared to TEF1- $\alpha$ with 0.021 typing score. This allelic profile result was supported by phylogenetic network parallelograms based on neighbor net algorithm, which showed genetic diversity in studied isolates though some were sharing characters. This relationship study was further supported by generating phylogram of concatenated dataset by applying K2 evolutionary model in maximum likelihood phylogenetic analysis, which resolved phylogenetic diversity and relatedness among these fungal isolates with maximum bootstrap support.

\section{REFERENCES}

[1] Al Adawi, A. O., Barnes, I., Khan, I. A., A Subhi, A. M. A., Jahwari, A. A., Deadman, M. L., Wingfield, B. D., Wingfield, M. J. (2013): Ceratocystis manginecans associated with a serious wilt disease of two native legume trees in Oman and Pakistan. Australasian Plant Pathology 42(2): 179-193.

[2] Baker, C. J., Harrington, T. C., Krauss, U., Alfenas, A. C. (2003): Genetic variability and host specialization in the Latin American clade of Ceratocystis fimbriata. Phytopathology 93(10): 1274-1284. 
[3] Fourie, A., Wingfield, M. J., Wingfield, B. D., Barnes, I. (2015): Molecular markers delimit cryptic species in Ceratocystis sensu stricto. - Mycological Progress 14(1): 1020.

[4] Halsted, B. D. (1890): Some fungus diseases of the sweet potato. - New Jersey Agricultural College Experiment Station Bulletin (76): 25-27.

[5] Harrington, T. C. (2000): Host specialization and speciation in the American wilt pathogen. - Fitopatologia Brasileira 25: 262-263.

[6] Heath, R. N., Wingfield, M. J., Wingfield, B. D., Meke, G., Mbaga, A., Roux, J. (2009): Ceratocystis species on Acacia mearnsii and Eucalyptus spp. in eastern and southern Africa including six new species. - Fungal Diversity 34: 41-67.

[7] Johnson, J. A., Harrington, T. C., Engelbrecht, C. J. B. (2005): Phylogeny and taxonomy of the North American clade of the Ceratocystis fimbriata complex. - Mycologia 97(5): 1067-1092.

[8] Li, Q., Harrington, T. C., McNew, D., Li, J. (2017): Ceratocystis uchidae, a new species on Araceae in Hawaii and Fiji. - Mycoscience 58(6): 398-412.

[9] Marin, M., Castro, B., Gaitan, A., Preisig, O., Wingfield, B. D., Wingfield, M. J. (2003): Relationships of Ceratocystis fimbriata isolates from Colombian coffee-growing regions based on molecular data and pathogenicity. - Journal of Phytopathology 151(7-8): 395405.

[10] Oliveira, L. S., Harrington, T. C., Ferreira, M. A., Damacena, M. B., Al-Sadi, A. M., AlMahmooli, I. H., Alfenas, A. C. (2015): Species or genotypes? Reassessment of four recently described species of the Ceratocystis wilt pathogen, Ceratocystis fimbriata, on Mangifera indica. - Phytopathology 105(9): 1229-1244.

[11] Seifert, K. A., Wingfield, M. J., Kendrick, W. B. (1993): A nomenclator for Described Species of Ceratocystis, Ophiostoma, Ceratocystiopsis, Ceratostomella and Sphaeronaemella. - American Phytopathological Society, St. Paul, Minnesota, pp. 269287.

[12] Tamura, K., Stecher, G., Peterson, D., Filipski, A., Kumar, S. (2013): MEGA6: molecular evolutionary genetics analysis version 6.0. - Molecular Biology and Evolution 30(12): 2725-2729.

[13] Van Wyk, M., Al-Adawi, A. O., Wingfield, B. D., A-Subhi, A. M., Deadman, M. L., Wingfield, M. J. (2005): DNA based characterization of Ceratocystis fimbriata isolates associated with mango decline in Oman. - Australasian Plant Pathology. 34(4): 587-590.

[14] White, T. J., Bruns, T., Lee, S. J. W. T., Taylor, J. L. (1990): Amplification and Direct Sequencing of Fungal Ribosomal RNA Genes for Phylogenetics. - In: Innis, M. A., Gelfand, D. H., Sninsky, J. J., White, T. J. (eds.) PCR Protocols. A Guide to Methods and Applications. Academic Press, San Diego, pp. 315-322.

[15] Wingfield, M. J., D Beer, C., Visser, C., Wingfield, B. D. (1996): A new Ceratocystis species defined using morphological and ribosomal DNA sequence comparisons. Systematic and Applied Microbiology 19(2): 191-202. 